\title{
氟烷磺酰氟和全乙酰基保护的吡喃型半缩醛单糖的氟代反应
}

\author{
严兆华 $*, a, b$ 赖焜民 ${ }^{a}$ 田伟生 ${ }^{*, b}$ 余章昕 ${ }^{a}$ \\ $\left({ }^{a}\right.$ 南昌大学化学系 南昌 330031) \\ ( $b$ 中国科学院上海有机化学研究所 上海 200032)
}

\begin{abstract}
摘要 考察了 5-氢-3-氧杂-1,1,2,2,4,4,5,5-八氟戊烷磺酰氟 $\left(\mathrm{HCF}_{2} \mathrm{CF}_{2} \mathrm{OCF}_{2} \mathrm{CF}_{2} \mathrm{SO}_{2} \mathrm{~F}\right)$ 和全乙酰基保护的吡喃型半缩醛单 糖在有机碱存在下的氟代反应. 实验结果表明, 氟烷基磺酰氟试剂 $\mathrm{HCF}_{2} \mathrm{CF}_{2} \mathrm{OCF}_{2} \mathrm{CF}_{2} \mathrm{SO}_{2} \mathrm{~F}$ 能够诱导全乙酰基保护的吡 喃型半缩醛单糖发生氟代反应而生成相应的氟代糖苷，得率 $44 \% \sim 94 \%$. 该方法具有反应条件温和和后处理简便等优 点，可作为一种有效的方法用于氟代糖苷的制备中.
\end{abstract}

关键词 5-氢-3-氧杂-1,1,2,2,4,4,5,5-八氟戊烷磺酰氟；吡喃型半缩醛单糖；氟代糖苷；氟代反应

\section{Deoxyfluorination of Peracetylated Pyranose Hemiacetal with Fluoroalkanesulfonyl Fluoride}

\author{
Yan, Zhaohua ${ }^{*, a, b} \quad$ Lai, Kunmin $^{a} \quad$ Tian, Weisheng ${ }^{*, b} \quad$ Yu, Zhangxin ${ }^{a}$ \\ ( ${ }^{a}$ Department of Chemistry, Nanchang University, Nanchang 330031) \\ ( ${ }^{b}$ Shanghai Institute of Organic Chemistry, Shanghai 200032)
}

\begin{abstract}
The deoxyfluorination of peracetylated pyranose hemiacetals with 5- $H$-3-oxa-1,1,2,2,4,4,5,5octafluoropentanesulfonyl fluoride $\left(\mathrm{HCF}_{2} \mathrm{CF}_{2} \mathrm{OCF}_{2} \mathrm{CF}_{2} \mathrm{SO}_{2} \mathrm{~F}\right)$ in the presence of an organic base was investigated resulting in the smooth formation of corresponding glycosyl fluorides in the yield of $44 \% \sim 94 \%$. The mild reaction conditions and easy work-up procedure are the two advantages for this methodology and it can be used as an alternative method for the preparation of glycosyl fluorides.
\end{abstract}

Keywords 5-H-3-oxa-1,1,2,2,4,4,5,5-octafluoropentanesulfonyl fluoride; pyranose hemiacetal; glycosyl fluoride; deoxyfluorination

\section{1 引言}

氟代糖苷是近三十几年来才引起人们浓厚兴趣并 得到较大发展的一类糖化合物. 早期的研究发现, 有些 氟代糖苷可以作为良好的酶抑制剂 ${ }^{[1]}$. 由于 $\mathrm{C}-\mathrm{F}$ 键比 $\mathrm{C}-\mathrm{Cl}$ 键或 $\mathrm{C}-\mathrm{Br}$ 键具有更高的化学键能 ${ }^{[2]}$, 和相应的 氯代糖苷或溴代糖苷相比, 氟代糖苷具有更高的稳定 性. 因其稳定性更高, 使用上更方便和立体选择性更好, 近年来氟代糖苷常被用来取代氯代糖苷或溴代糖苷而 应用于糖苷化反应中. 氟代糖苷是一种很好的糖供体, 它不仅能通过 $\mathrm{C}-\mathrm{O}$ 键形成糖苷, 而且能通过 $\mathrm{C}-\mathrm{N}$, $\mathrm{C}-\mathrm{S}$ 和 $\mathrm{C}-\mathrm{C}$ 键形成糖苷, 因此在糖化学合成中具有十
分重要的作用 ${ }^{[3]}$. 文献上报道的制备氟代糖苷的方法有 多种，现简要归纳如下. 1984 年 Hayashi 等 ${ }^{[4]}$ 报道了吡啶 和氟化氢的复合物与 1-乙酰化的糖化合物作用可转化 为氟代糖苷. 此后二乙胺基三氟化硫 $\left(\mathrm{Et}_{2} \mathrm{NSF}_{3}\right.$, DAST 试 剂)被发现和半缩醛糖化合物反应可用来制备氟代糖 苷 ${ }^{[5]} . \mathrm{Et}_{2} \mathrm{NSF}_{3} / N$-溴代丁二酰亚胺 ${ }^{[6]}$ 或高价碘化合物 ${ }^{\left[{ }^{[7]}\right.}$ 如 $\mathrm{ArIF}_{2}$ 能够将硫代糖苷转化为氟代糖苷. 最近 Hara 等 ${ }^{[8]}$ 报道了 $m-\mathrm{CH}_{3} \mathrm{PhCF}_{2} \mathrm{NEt}_{2}$ 可用于诱导半缩醛糖化合物生 成氟代糖苷. 除此之外, $n-\mathrm{Bu}_{4} \mathrm{NF}, \mathrm{AgF}, \mathrm{ZnF}_{2}$ 和 $\mathrm{PPh}_{3} / \mathrm{EtO}_{2} \mathrm{C}-\mathrm{N}=\mathrm{N}-\mathrm{CO}_{2} \mathrm{Et}^{-} / \mathrm{Et}_{3} \mathrm{O}^{+} \mathrm{BF}_{4}^{-}$等试剂也常被用于 制备氟代糖苷.

\footnotetext{
* E-mail: yanzh@ncu.edu.cn; wstian@sioc.ac.cn

Received December 16, 2011; accepted April 23, 2012.
} 
另一方面, 已有的研究表明, 氟烷磺酰氟 (如 $n-\mathrm{C}_{4} \mathrm{~F}_{9} \mathrm{SO}_{2} \mathrm{~F}, n-\mathrm{C}_{8} \mathrm{~F}_{17} \mathrm{SO}_{2} \mathrm{~F}$ 和 $\mathrm{HCF}_{2} \mathrm{CF}_{2} \mathrm{OCF}_{2} \mathrm{CF}_{2} \mathrm{SO}_{2} \mathrm{~F}$ 等, 以 $\mathrm{R}_{\mathrm{f}} \mathrm{SO}_{2} \mathrm{~F}$ 表示, 下同)是一类很有应用潜力的有机合成 试剂. 1995 年, 德国的 Vorbruggen 等 ${ }^{[9]}$ 首次报道了 $\mathrm{R}_{\mathrm{f}} \mathrm{SO}_{2} \mathrm{~F}$ 和醇反应能生成单氟代化合物. 1996年我们课题 组 ${ }^{[10]}$ 报道了 $\mathrm{R}_{\mathrm{f}} \mathrm{SO}_{2} \mathrm{~F}$ 和甾体-3-酮的烯醇磺酸酯化反应并 成功地将该方法应用于甾体药物爱普列特的研究开发 和生产中. 此后我们课题组又相继报道了 $\mathrm{R}_{\mathrm{f}} \mathrm{SO}_{2} \mathrm{~F}$ 和 19羟基睪酮的高烯丙位重排反应 ${ }^{[11]}$ 及应用于海洋天然产 物 spiniferin $1^{[12]}$ 和新型雌激素拮抗剂 ${ }^{[13]}$ 的全合成中; 和 赤式邻位二醇的反应制备顺式环氧化合物并应用于桥 环黄皮酰胺的全合成 ${ }^{[14]}$; 和烯烃作用生成环氧化合物 的反应 ${ }^{[15]}$; 作为缩合试剂诱导羧酸与醇和胺的酯化反 应和酰胺化反应生成羧酸酯和酰胺等 ${ }^{[16]}$. 在我们所考 察的这些反应中, $\mathrm{R}_{\mathrm{f}} \mathrm{SO}_{2} \mathrm{~F}$ 不仅作为活化试剂参与到反应 中, 而且反应后所生成的产物还是一种高附加值工业商 品含氟表面活性剂氟烷基磺酸盐, 因此是典型的 “共生 反应” ${ }^{[17]}$. 然而到目前为止, 还未见有报道过 $\mathrm{R}_{\mathrm{f}} \mathrm{SO}_{2} \mathrm{~F}$ 和 半缩醛糖化合物的氟代反应. 为了继续探讨 $\mathrm{R}_{\mathrm{f}} \mathrm{SO}_{2} \mathrm{~F}$ 试 剂在有机合成化学中的应用, 我们着手考察了 $\mathrm{R}_{\mathrm{f}} \mathrm{SO}_{2} \mathrm{~F}$ 和分子结构较为简单的全乙酰基保护的吡喃型半缩醛 单糖(1)的反应. 我们设想糖分子异头碳上的半缩醛羟 基在碱存在下将首先和 $\mathrm{R}_{\mathrm{f}} \mathrm{SO}_{2} \mathrm{~F}$ 反应而生成离去性能很 强的氟烷磺酸酯, 接着溶液中共存的氟负离子将进攻异 头碳而生成氟代糖苷(2 和 3). 见 Eq. 1.

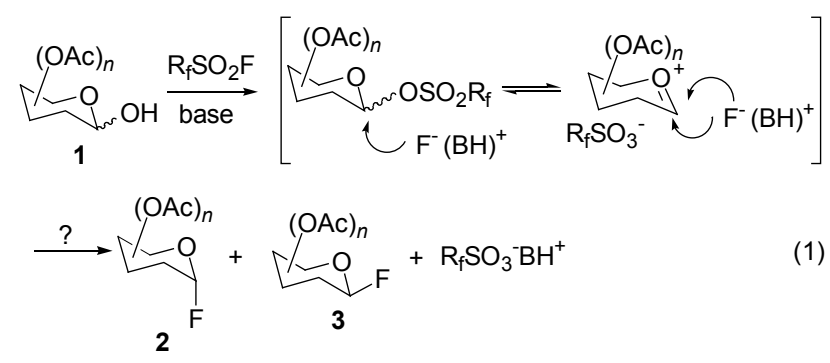

从 Eq. 1 可以看出, 反应后除了可能生成预料的产 物氟代糖苷外, 还可能同时生成有用的氟烷基磺酸盐. 为了验证这一设想, 我们探索性地研究了 5 个全乙酰基 保护的吡喃型半缩醛单糖和手头上易得的氟烷磺酰氟 试剂5-氢-3-氧杂-1, 1,2,2,4,4,5,5-八氟戊烷磺酰氟 $\left(\mathrm{HCF}_{2} \mathrm{CF}_{2} \mathrm{OCF}_{2} \mathrm{CF}_{2} \mathrm{SO}_{2} \mathrm{~F}\right)$ 的反应. 结果证实了我们的设 想, 反应确实是生成了相应的氟代糖苷 $(2$ 和 3$)$.

\section{2 结果与讨论}

\section{1 反应条件的优化}

首先, 我们以 2,3,4-三乙酰基- $D$-吡喃型木糖(1a)为
底物, 考察了在不同溶剂和碱存在下与 $\mathrm{HCF}_{2} \mathrm{CF}_{2} \mathrm{OCF}_{2}-$ $\mathrm{CF}_{2} \mathrm{SO}_{2} \mathrm{~F}$ (在 Eq. 2 和表 1 中以 $\mathrm{R}_{\mathrm{f}} \mathrm{SO}_{2} \mathrm{~F}$ 表示)的反应情况, 见 Eq. 2.

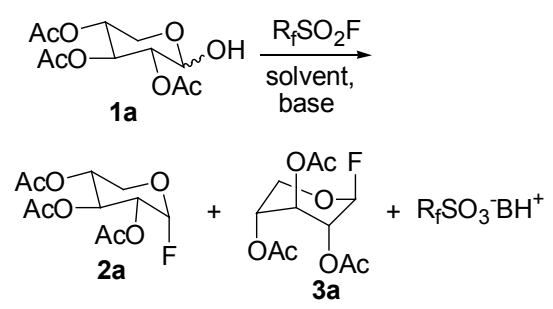

实验结果见表 1 . 从表 1 的反应结果可以看出, 溶 剂和碱对该反应影响很大. 当使用 $\mathrm{DBU}$ 作碱时, 若以 $\mathrm{CH}_{2} \mathrm{Cl}_{2}$ 作溶剂, 原料只有少量转化, 延长反应时间和升 高反应温度对反应结果的影响都不大. 以 $\mathrm{DME}$ (乙二醇 二甲醚)作溶剂时也是如此. 其它溶剂如 THF, PhMe, $\mathrm{MeCN}$ 和二氧六环都适合于此反应，反应 $10 \mathrm{~min}$ 后，原 料都转化完全, 且生成了两个主要产物, 它们分别是 $\alpha$ 体和 $\beta$ 体的氟代糖苷. 这两个产物的 ${ }^{1} \mathrm{H}$ NMR 谱图和 ${ }^{19} \mathrm{~F}$ NMR 谱图同文献 ${ }^{[18]}$ 的都一致. 我们同时还发现, 当 1a: $\mathrm{R}_{\mathrm{f}} \mathrm{SO}_{2} \mathrm{~F}: \mathrm{DBU}=1: 2: 2$ (摩尔当量比)时, 反应 10 $\min$ 后，原料只有部分转化，同样延长反应时间和升高 反应温度, 反应依然如此(Entry 7 in Table 1). 即使将三 者比例提高到 $1: 5: 5$, 仍有少部分原料末被转化 (Entry 8 in Table 1). 但当比例提高到 $1: 7: 7$ 时, 反应 $10 \mathrm{~min}$ 后, 原料即完全转化. 该反应需要 7 摩尔当量的 $\mathrm{R}_{\mathrm{f}} \mathrm{SO}_{2} \mathrm{~F}$ 试剂和碱说明半缩醛 1 和 $\mathrm{R}_{\mathrm{f}} \mathrm{SO}_{2} \mathrm{~F}$ 之间的反应存 在着较大的位阻. 这可能是由于 $\mathrm{R}_{\mathrm{f}} \mathrm{SO}_{2} \mathrm{~F}$ 和 $\mathrm{DBU}$ 都是体 积比较大的分子; 此外, 底物 1 属于仲醇, 且环上还有

表 1 溶剂和碱对 $1 \mathrm{a}$ 和 $\mathrm{R}_{\mathrm{f}} \mathrm{SO}_{2} \mathrm{~F}$ 的反应的影响 ${ }^{a}$

Table 1 The effect of solvents and bases on deoxyfluorination of $1 \mathrm{a}$ and $\mathrm{R}_{\mathrm{f}} \mathrm{SO}_{2} \mathrm{~F}$

\begin{tabular}{|c|c|c|c|c|c|}
\hline \multirow{2}{*}{ Entry } & \multirow{2}{*}{ Base } & \multirow{2}{*}{ Solvent } & \multicolumn{3}{|c|}{ Isolated yield $/ \%$} \\
\hline & & & $1 \mathrm{a}$ & $2 a$ & $3 \mathbf{a}$ \\
\hline 1 & DBU & $\mathrm{CH}_{2} \mathrm{Cl}_{2}$ & 76 & 10 & 5 \\
\hline 2 & DBU & $\mathrm{PhMe}$ & 0 & 12 & 72 \\
\hline 3 & DBU & $\mathrm{MeCN}$ & 0 & 15 & 55 \\
\hline 4 & DBU & Dioxane & 0 & 16 & 60 \\
\hline 5 & DBU & THF & 0 & 35 & 59 \\
\hline 6 & $\mathrm{DBU}$ & DME & 70 & 8 & 6 \\
\hline $7^{b}$ & DBU & THF & 60 & 15 & 18 \\
\hline $8^{c}$ & DBU & THF & 20 & 30 & 42 \\
\hline 9 & $\mathrm{Et}_{3} \mathrm{~N}$ & THF & 100 & 0 & 0 \\
\hline 10 & $\mathrm{KOH}$ & THF & 100 & 0 & 0 \\
\hline
\end{tabular}


多个体积较大的 OAc 基团, 这些因素都会对反应带来 较大的位阻. 从表 1 还可看出, 碱性较弱的有机碱 $\mathrm{Et}_{3} \mathrm{~N}$ 和无机碱 $\mathrm{KOH}$ 在室温下对该反应都不适用, 即使延长 反应时间和升高反应温度, 情况仍如此, 由此可见, 反 应时间和温度对该氟苷化反应影响不大. 我们也使用了 $n-\mathrm{C}_{4} \mathrm{~F}_{9} \mathrm{SO}_{2} \mathrm{~F}$ 和 $n-\mathrm{C}_{8} \mathrm{~F}_{17} \mathrm{SO}_{2} \mathrm{~F}$ 分别代替 $\mathrm{HCF}_{2} \mathrm{CF}_{2} \mathrm{OCF}_{2} \mathrm{CF}_{2}$ $\mathrm{SO}_{2} \mathrm{~F}$ 用于 Eq. 2 的反应, 实验结果大体上一致.

文献报道 ${ }^{[19]}, \beta$ 体产物 3a 的分子构型中, 取代基 $\mathrm{F}$ 和 $\mathrm{OAc}$ 都不处在 $\mathrm{e}$ 键, 而是全部处于 $\mathrm{a}$ 键, 这是通过该 化合物的 ${ }^{1} \mathrm{H}$ NMR 谱图中出现的 ${ }^{3} J_{\mathrm{H}-\mathrm{H}}$ 偶合常数的大小 决定的.

\section{2 其它底物的反应情况}

在考察了 1a 和 $\mathrm{HCF}_{2} \mathrm{CF}_{2} \mathrm{OCF}_{2} \mathrm{CF}_{2} \mathrm{SO}_{2} \mathrm{~F}$ 的反应后, 我们进一步探讨了另外 4 个全保护的吡喃型半缩醛糖 $1 \mathrm{~b}, 1 \mathrm{c}, 1 \mathrm{~d}$ 和 $1 \mathrm{e}$ 的氟苷化反应, 实验结果见表 2 . 从表 2 结果可知, $1 \mathrm{~b}$ 和 $1 \mathrm{c}$ 的氟苷化反应只生成了 $\beta$ 体产物, 其 中 $\alpha$ 体产物极少. 这可能是由于 C-2 位上的 OAc 取代基 处于 $\mathrm{e}$ 键而引起了 “邻位参与” 作用, 使得氟负离子只 能从 $\beta$ 面进攻的结果, 见 Scheme 1. 虽然 $1 \mathbf{a}$ 分子中 C-2 位的 OAc 基团也处于 $\mathrm{e}$ 键上, 然而也生成了一定比例的 $\alpha$ 体产物, 而不象 $1 \mathbf{b}$ 和 $1 \mathrm{c}$ 的氟苷化反应那样只生成了 $\beta$ 体产物, 我们对这一反应现象差异的解释是: 1a 分子中 $\mathrm{C}-5$ 位没有 $\mathrm{CH}_{2} \mathrm{OAc}$ 基团, 因此位阻作用小而导致氟苷 化反应速度比较快, 在 $\mathrm{C}-2$ 位处于 $\mathrm{e}$ 键上的 $\mathrm{OAc}$ 基团引 起 “邻位参与” 作用之前, 溶液中共存的 $[\mathrm{DBUH}]^{+} \mathrm{F}^{-}$即 可从 $\alpha$ 面进攻异头碳而生成 $\alpha$ 体产物; 而 $1 \mathrm{~b}$ 和 $1 \mathrm{c}$ 分子 的 C-5 位有 $\mathrm{CH}_{2} \mathrm{OAc}$ 基团, 由于位阻作用使得氟苷化反 应速度更慢，导致 $\mathrm{C}-2$ 位处于 $\mathrm{e}$ 键上的 $\mathrm{OAc}$ 基团有足够 的时间引起 “邻位参与” 作用, 因而抑制了 $[\mathrm{DBUH}]^{+} \mathrm{F}^{-}$ 从 $\alpha$ 面进攻异头碳而没有生成 $\alpha$ 体产物.

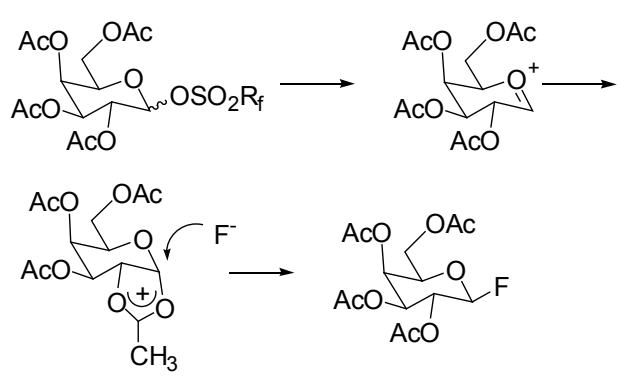

Scheme 1

从表 2 还可看出, $1 \mathrm{~d}$ 和 $1 \mathrm{e}$ 分子中 $\mathrm{C}-2$ 位 $\mathrm{OAc}$ 基团 处于 $\mathrm{a}$ 键上. 由于 $\mathrm{a}$ 键上的 $O A c$ 基团与吡喃环上的氧鎓 中心在立体上所处的相对位置不容易产生 “邻位参与” 作用, 因此处于 $\mathrm{a}$ 键上的 OAc 基团所引起的“邻位参与” 作用不明显, 因而产物中都含有相当量的 $\alpha$ 体氟代糖苷 产物. 此外, 表 2 中所使用的 4 个底物的反应原料都没
转化完全, 即使增加 $\mathrm{R}_{\mathrm{f}} \mathrm{SO}_{2} \mathrm{~F}$ 和 $\mathrm{DBU}$ 的量或者改变溶 剂、反应温度和反应时间, 效果都不明显. 我们也尝试 过在反应体系中额外加入氟化钾或四丁基氟化铵期待 能促进原料转化完全, 然而实验结果并不理想. 这可能 是由于表 2 中 4 个底物分子的 C-5 位存在的 $\mathrm{CH}_{3}$ 或 $\mathrm{CH}_{2} \mathrm{OAc}$ 基团对氟苷化反应有较大的位阻作用的缘故. 最后还需指出的是, 4 个底物所使用的溶剂也不同, 比 如, 对 $1 \mathrm{~b}$ 的反应, 使用 $\mathrm{CH}_{2} \mathrm{Cl}_{2}$ 最好, 而 $1 \mathrm{e}$ 的反应在 $\mathrm{MeCN}$ 中最好.

表 2 中的各个产物都是已知化合物，它们的 ${ }^{1} \mathrm{H}$ NMR 谱图和 ${ }^{19} \mathrm{~F}$ NMR 谱图在文献中均有报道 ${ }^{[20,21]}$. 我 们所得产物的谱图与文献值相符.

\section{3 结论}

以 $\mathrm{DBU}$ 为碱, $\mathrm{HCF}_{2} \mathrm{CF}_{2} \mathrm{OCF}_{2} \mathrm{CF}_{2} \mathrm{SO}_{2} \mathrm{~F}$ 能诱导全乙酰 基保护的吡喃型半缩醛单糖发生氟苷化反应生成相应 的氟代糖苷，收率中等到优秀. 其中若全乙酰基保护的 吡喃型半缩醛单糖的 C-5 位上没有取代基时, 原料能转 化完全; 若 C-5 位上有取代基(如 $\mathrm{CH}_{3}$ 或 $\mathrm{CH}_{2} \mathrm{OAc}$ ), 则原 料通常转化不完全. 在所考察的 5 个底物中, $2,3,4,6$-四 乙酰基- $D$-吡喃型葡萄糖和 2,3,4,6-四乙酰基- $D$-吡喃型 半乳糖同 $\mathrm{HCF}_{2} \mathrm{CF}_{2} \mathrm{OCF}_{2} \mathrm{CF}_{2} \mathrm{SO}_{2} \mathrm{~F}$ 的反应只生成 $\beta$ 型氟代 糖苷; $2,3,4,6$-四乙酰基- $D$-吡喃型甘露糖、2,3,4,-三乙酰 基- $L$-吡喃型鼠李糖和 2,3,4,-三乙酰基- $D$-吡喃型木糖同 $\mathrm{HCF}_{2} \mathrm{CF}_{2} \mathrm{OCF}_{2} \mathrm{CF}_{2} \mathrm{SO}_{2} \mathrm{~F}$ 的反应产物中既有 $\beta$ 型氟代糖 苷，又有 $\alpha$ 型氟代糖苷. 本工作为合成全保护的氟代糖 苷提供了一种新的方法, 并且该方法还具有反应条件温 和和后处理简单等优点.

\section{4 实验部分}

\section{1 试剂与仪器}

所使用的五个底物全乙酰基保护的吡喃型半缩醛 单糖都是自己合成的, 即以吡喃型半缩醛单糖为原料首 先和乙酸䣶反应得到全保护的化合物, 再经氨选择性水 解而制得; 所使用的 5-氢-3-氧杂- $1,1,2,2,4,4,5,5$-八氟戊 烷磺酰氟 $\left(\mathrm{HCF}_{2} \mathrm{CF}_{2} \mathrm{OCF}_{2} \mathrm{CF}_{2} \mathrm{SO}_{2} \mathrm{~F}\right)$ 是通过 5-碘-3-氧杂$1,1,2,2,4,4,5,5$-八氟戊烷磺酰氟 $\left(\mathrm{ICF}_{2} \mathrm{CF}_{2} \mathrm{OCF}_{2} \mathrm{CF}_{2} \mathrm{SO}_{2} \mathrm{~F}\right)$ 和锌粉在二氧六环中发生还原反应而制备得到; ${ }^{1} \mathrm{H}$ NMR 用 Bruker-300 (300 MHz)核磁共振仪(四甲基硅烷 作内标, $\mathrm{CDCl}_{3}$ 作溶剂); ${ }^{19} \mathrm{~F}$ NMR 用 Bruker-300 (282 $\mathrm{MHz})$ 核磁共振仪 $\left(\mathrm{CF}_{3} \mathrm{COOH}\right.$ 作内标, 化学位移高场为 正); 使用的其它试剂均为分析纯, 都未经任何后处理 就直接用于反应中; 柱层析所用吸附剂为青岛海洋化工 厂生产的硅胶 $\mathrm{H}(10 \sim 40 \mu, 200 \sim 300$ 目); 洗脱剂为不 
表 $21 \mathrm{~b}, 1 \mathrm{c}, 1 \mathrm{~d}$ 和 $1 \mathrm{e}$ 与 $\mathrm{R}_{\mathrm{f}} \mathrm{SO}_{2} \mathrm{~F}$ 的氟苷化反应结果 ${ }^{a}$

Table 2 Deoxyfluorination of $\mathbf{1 b}, \mathbf{1 c}, \mathbf{1 d}$ and $1 \mathrm{e}$ with $\mathrm{R}_{\mathrm{f}} \mathrm{SO}_{2} \mathrm{~F}$

Entry

${ }^{a} 1 \mathrm{a}: \mathrm{RfSO}_{2} \mathrm{~F}:$ base $=1: 7: 7$ (molar ratio). And all the reactions were run at $10{ }^{\circ} \mathrm{C}$ and the reaction time is $10 \mathrm{~min}$.

同体积比例的石油醚(沸程为 $60 \sim 90{ }^{\circ} \mathrm{C}$ ) 和乙酸乙酯的 混合溶剂; 用于反应的溶剂均经过重蒸处理; 石油醚, 乙酸乙酯, 二氯甲烷和甲醇等柱层析和后处理用溶剂均 为市售分析纯化学试剂.

\section{2 氟烷磺酰氟 $\mathrm{HCF}_{2} \mathrm{CF}_{2} \mathrm{OCF}_{2} \mathrm{CF}_{2} \mathrm{SO}_{2} \mathrm{~F}$ 与全乙酰基} 保护的吡喃型半缩醛单糖(1)的反应

典型实验步骤如下:

$10{ }^{\circ} \mathrm{C}$ 下, 将 $0.2 \mathrm{mmol}$ 的全乙酰基保护的吡喃型半 缩醛单糖 1 溶解于 $3 \mathrm{~mL}$ 溶剂中，接着加入 $1.4 \mathrm{mmol} 1,8-$ 二氮杂双环 $[5,4,0]$ 十一-7-烯(DBU), 最后慢慢经由针筒 滴入 $1.4 \mathrm{mmol} 5$-氢-3-氧杂-1,1,2,2,4,4,5,5-八氟戊烷磺酰 氟 $\left(\mathrm{HCF}_{2} \mathrm{CF}_{2} \mathrm{OCF}_{2} \mathrm{CF}_{2} \mathrm{SO}_{2} \mathrm{~F}\right)$, 并继续在 $10{ }^{\circ} \mathrm{C}$ 下电磁搅拌 一定的时间. 浓缩除去溶剂, 残留物经硅胶快速柱层析 分离纯化. 用石油醚和乙酸乙酯的混合溶剂作洗脱剂淋 洗. 分别得纯品 $\alpha$ 型氟代糖苷化合物 $\mathbf{2}$ 和 $\beta$ 型氟代糖苷 化合物 3.

2,3,4-O-三乙酰基- $\alpha$ - $D$-吡喃型氟代木糖苷(2a)：得 率 35\%, 无色固体; m.p. 56 58 ${ }^{\circ} \mathrm{C} ;{ }^{1} \mathrm{H}$ NMR $\left(\mathrm{CDCl}_{3}\right.$, $300 \mathrm{MHz}) \delta: 2.05$ (s, 3H, $\left.\mathrm{COCH}_{3}\right), 2.07$ (s, 3H, $\left.\mathrm{COCH}_{3}\right)$, $2.10\left(\mathrm{~s}, 3 \mathrm{H}, \mathrm{COCH}_{3}\right), 3.79$ (t, $\left.J=11.0 \mathrm{~Hz}, 1 \mathrm{H}, \mathrm{H}-5 \alpha\right), 3.98$ (dd, $J=11.0,2.8 \mathrm{~Hz}, 1 \mathrm{H}, \mathrm{H}-5 \beta$ ), 4.88 (ddd, $J=24.0,10.0$, $2.8 \mathrm{~Hz}, 1 \mathrm{H}, \mathrm{H}-2), 5.01 \sim 5.05$ (m, 1H, H-4), 5.45 (t, $J=$ $10.0 \mathrm{~Hz}, 1 \mathrm{H}, \mathrm{H}-3), 5.68$ (dd, $J=53.0,2.7 \mathrm{~Hz}, 1 \mathrm{H}, \mathrm{H}-1) ;{ }^{19} \mathrm{~F}$ NMR (282 MHz, $\mathrm{CDCl}_{3}, \mathrm{CF}_{3} \mathrm{COOH}$ 作内标) $\delta:-74.5$ (dd, $J=53.0,24.0 \mathrm{~Hz}, 1 \mathrm{~F}, \mathrm{~F}-1$ ); IR (KBr) v: 2951, 1758,
$1741,1372,1253,1228,1056 \mathrm{~cm}^{-1}$; EI-MS $m / z: 43$ (100.00), 258 (3.24), $278\left(\mathrm{M}^{+}, 0.10\right)$.

$2,3,4-O$-三乙酰基- $\beta$ - $D$-吡喃型氟代木糖苷(3a): 得 率 59\%, 无色固体; m.p. $50 \sim 51{ }^{\circ} \mathrm{C} ;{ }^{1} \mathrm{H}$ NMR $\left(\mathrm{CDCl}_{3}\right.$, $300 \mathrm{MHz}) \delta: 2.03\left(\mathrm{~s}, 3 \mathrm{H}, \mathrm{COCH}_{3}\right), 2.07\left(\mathrm{~s}, 3 \mathrm{H}, \mathrm{COCH}_{3}\right)$, $2.20\left(\mathrm{~s}, 3 \mathrm{H}, \mathrm{COCH}_{3}\right), 3.78(\mathrm{dd}, J=13.0,3.2 \mathrm{~Hz}, 1 \mathrm{H}$, H-5 $\alpha$ ), 4.29 (dd, $J=13.0,2.8 \mathrm{~Hz}, 1 \mathrm{H}, \mathrm{H}-5 \beta$ ), 4.84 (dd, $J=$ 7.5, $3.4 \mathrm{~Hz}, 1 \mathrm{H}, \mathrm{H}-2), 4.91 \sim 4.96(\mathrm{~m}, 1 \mathrm{H}, \mathrm{H}-4), 5.06(\mathrm{t}$, $J=7.6 \mathrm{~Hz}, 1 \mathrm{H}, \mathrm{H}-3), 5.45$ (dd, $J=50.1,2.7 \mathrm{~Hz}, 1 \mathrm{H}, \mathrm{H}-1$ ); ${ }^{19} \mathrm{~F}$ NMR $\left(\mathrm{CDCl}_{3}, 282 \mathrm{MHz}, \mathrm{CF}_{3} \mathrm{COOH}\right.$ 作内标 $) \delta$ : $-59.6(\mathrm{dd}, J=50.2,7.3 \mathrm{~Hz}, 1 \mathrm{~F}, \mathrm{~F}-1)$; IR $(\mathrm{KBr}) v: 2973$, 2937, 1759, 1374, 1253, 1219, $1047 \mathrm{~cm}^{-1}$; EI-MS m/z: 43 (100.00), 258 (6.58), $278\left(\mathrm{M}^{+}, 0.09\right)$.

$2,3,4,6-O$-四乙酰基- $\beta$ - $D$-吡喃型氟代葡萄糖苷 $(\mathbf{3 b})$ : 得率 $52 \%$, 无色油状物; ${ }^{1} \mathrm{H}$ NMR $\left(\mathrm{CD}_{3} \mathrm{COCD}_{3}, 300\right.$ $\mathrm{MHz}) \delta: 1.95$ (s, $\left.3 \mathrm{H}, \mathrm{COCH}_{3}\right), 1.98\left(\mathrm{~s}, 3 \mathrm{H}, \mathrm{COCH}_{3}\right), 2.00$ (s, $\left.3 \mathrm{H}, \mathrm{COCH}_{3}\right), 2.05\left(\mathrm{~s}, 3 \mathrm{H}, \mathrm{COCH}_{3}\right), 4.06 \sim 4.09(\mathrm{~m}, 1 \mathrm{H}$, H-5), $4.12 \sim 4.17$ (m, 2H, H-6), $4.98 \sim 5.02(\mathrm{~m}, 1 \mathrm{H}, \mathrm{H}-2)$, 5.15 (t, $J=9.4 \mathrm{~Hz}, 1 \mathrm{H}, \mathrm{H}-4), 5.38$ (t, $J=9.4 \mathrm{~Hz}, 1 \mathrm{H}, \mathrm{H}-3$ ), $5.60(\mathrm{dd}, J=52.5,6.6 \mathrm{~Hz}, 1 \mathrm{H}, \mathrm{H}-1)$; EI-MS $m / z: 43$ (100.00), 169 (22.69), 330 (17.46), $350\left(\mathrm{M}^{+}, 0.17\right)$.

$2,3,4,6-O$-四乙酰基- $\beta$ - $D$-吡喃型氟代半乳糖苷 $(\mathbf{3 c})$ : 得率 $44 \%$, 无色油状物; ${ }^{1} \mathrm{H}$ NMR $\left(\mathrm{CDCl}_{3}, 300 \mathrm{MHz}\right) \delta$ : $2.01\left(\mathrm{~s}, 3 \mathrm{H}, \mathrm{COCH}_{3}\right), 2.05\left(\mathrm{~s}, 3 \mathrm{H}, \mathrm{COCH}_{3}\right), 2.13(\mathrm{~s}, 3 \mathrm{H}$, $\left.\mathrm{COCH}_{3}\right), 2.17\left(\mathrm{~s}, 3 \mathrm{H}, \mathrm{COCH}_{3}\right), 4.12 \sim 4.22(\mathrm{~m}, 3 \mathrm{H}, \mathrm{H}-5$, 
H-6), 5.06 (dd, $J=10.2,3.3 \mathrm{~Hz}, 1 \mathrm{H}, \mathrm{H}-3$ ), 5.28 (dd, $J=$ 49.4, $7.0 \mathrm{~Hz}, 1 \mathrm{H}, \mathrm{H}-1), 5.28 \sim 5.34(\mathrm{~m}, 1 \mathrm{H}, \mathrm{H}-2), 5.40 \sim$ $5.45(\mathrm{~m}, 1 \mathrm{H}, \mathrm{H}-4) ;{ }^{19} \mathrm{~F}$ NMR $\left(\mathrm{CDCl}_{3}, 282 \mathrm{MHz}\right.$, $\mathrm{CF}_{3} \mathrm{COOH}$ 作内标) $\delta:-64.5(\mathrm{dd}, J=54.5,9.3 \mathrm{~Hz}, 1 \mathrm{~F}$, F-1); IR (neat) v: 2980, 2942, 1755, 1435, 1373, 1221, 1170, 1149, $1066 \mathrm{~cm}^{-1}$; EI-MS m/z: 44 (100.00), 169 (24.42), $331\left[(\mathrm{M}-\mathrm{F})^{+}, 26.95\right]$.

2,3,4-O-三乙酰基- $\alpha-L$ - 吡喃型氟代鼠李糖苷(2d): 得率 35\%, 无色油状物; ${ }^{1} \mathrm{H} \mathrm{NMR}\left(\mathrm{CDCl}_{3}, 300 \mathrm{MHz}\right) \delta$ : 1.28 (d, $J=6.3 \mathrm{~Hz}, 3 \mathrm{H}, \mathrm{H}-6), 2.00$ (s, 3H, $\mathrm{COCH}_{3}$ ), 2.06 (s, $\left.3 \mathrm{H}, \mathrm{COCH}_{3}\right), 2.17\left(\mathrm{~s}, 3 \mathrm{H}, \mathrm{COCH}_{3}\right), 4.01 \sim 4.03(\mathrm{~m}, 3 \mathrm{H}$, H-5), 5.13 (t, $J=9.9 \mathrm{~Hz}, 1 \mathrm{H}, \mathrm{H}-4), 5.29$ (ddd, $J=10.1,3.5$, $1.8 \mathrm{~Hz}, 1 \mathrm{H}, \mathrm{H}-3), 5.36 \sim 5.41$ (m, 1H, H-2), 5.48 (dd, $J=$ $48.8,1.8 \mathrm{~Hz}, 1 \mathrm{H}, \mathrm{H}-1) ;{ }^{19} \mathrm{~F}$ NMR $\left(\mathrm{CDCl}_{3}, 282 \mathrm{MHz}\right.$, $\mathrm{CF}_{3} \mathrm{COOH}$ 作内标) $\delta:-60.4$ (d, $\left.J=51.4 \mathrm{~Hz}, 1 \mathrm{~F}, \mathrm{~F}-1\right)$; IR (neat) $v$ : 2989, 2943, 1757, 1373, 1245, 1222, 1179, 1059 $\mathrm{cm}^{-1}$; EI-MS m/z: 43 (100.00), 115 (9.40), 130 (10.02), 153 (12.33), 169 (6.85), 213 (3.54), 272 (13.14), $292\left(\mathrm{M}^{+}\right.$, $0.06)$.

2,3,4- $O$-三乙酰基- $\beta$ - $L$-吡喃型氟代鼠李糖苷(3d): 得率 $22 \%$, 无色油状物; ${ }^{1} \mathrm{H} \mathrm{NMR}\left(\mathrm{CDCl}_{3}, 300 \mathrm{MHz}\right) \delta$ : 1.25 (d, $J=6.5 \mathrm{~Hz}, 3 \mathrm{H}, \mathrm{H}-6), 1.95$ (s, 3H, $\left.\mathrm{COCH}_{3}\right), 2.08$ (s, $\left.3 \mathrm{H}, \mathrm{COCH}_{3}\right), 2.12\left(\mathrm{~s}, 3 \mathrm{H}, \mathrm{COCH}_{3}\right), 3.81 \sim 3.84(\mathrm{~m}, 1 \mathrm{H}$, H-5), 4.96 (t, $J=9.4 \mathrm{~Hz}, 1 \mathrm{H}, \mathrm{H}-4$ ), 5.15 (ddd, $J=9.7,3.9$, $0.9 \mathrm{~Hz}, 1 \mathrm{H}, \mathrm{H}-3), 5.50$ (dd, $J=3.4,1.1 \mathrm{~Hz}, 1 \mathrm{H}, \mathrm{H}-2), 5.73$ (dd, $J=48.2,1.1 \mathrm{~Hz}, 1 \mathrm{H}, \mathrm{H}-1) ;{ }^{19} \mathrm{~F}$ NMR $\left(\mathrm{CDCl}_{3}, 282\right.$ $\mathrm{MHz}, \mathrm{CF}_{3} \mathrm{COOH}$ 作内标) $\delta:-64.9$ (d, $J=47.9 \mathrm{~Hz}, 1 \mathrm{~F}$, F-1); IR (neat) $v: 2988,2943,1754,1646,1374,1253$, 1222, 1098, $1060 \mathrm{~cm}^{-1}$; EI-MS m/z: 44 (100.00), 273 $\left[(\mathrm{M}-\mathrm{F})^{+}, 0.86\right]$.

2,3,4,6-O-四乙酰基- $\alpha$ - $L$-吡喃型氟代甘露糖苷(2e): 得率 $23 \%$, 无色油状物; ${ }^{1} \mathrm{H} \mathrm{NMR}\left(\mathrm{CDCl}_{3}, 300 \mathrm{MHz}\right) \delta$ : $1.99\left(\mathrm{~s}, 3 \mathrm{H}, \mathrm{COCH}_{3}\right), 2.07$ (s, 3H, $\left.\mathrm{COCH}_{3}\right), 2.15(\mathrm{~s}, 3 \mathrm{H}$, $\left.\mathrm{COCH}_{3}\right), 2.16\left(\mathrm{~s}, 3 \mathrm{H}, \mathrm{COCH}_{3}\right), 4.15 \sim 4.32(\mathrm{~m}, 3 \mathrm{H}, \mathrm{H}-5$, H-6), $5.18 \sim 5.42$ (m, 3H, H-2, H-3, H-4), 5.55 (dd, $J=$ $48.4,1.5 \mathrm{~Hz}, 1 \mathrm{H}, \mathrm{H}-1) ;{ }^{19} \mathrm{~F}$ NMR $\left(\mathrm{CDCl}_{3}, 282 \mathrm{MHz}\right.$, $\mathrm{CF}_{3} \mathrm{COOH}$ 作内标) $\delta:-61.3(\mathrm{~d}, J=48.4 \mathrm{~Hz}, 1 \mathrm{~F}, \mathrm{~F}-1)$; IR (neat) $v: 2962,1752,1435,1371,1228,1178,1056 \mathrm{~cm}^{-1}$; EI-MS $m / z: 43$ (100.00), 109 (26.93), 169 (66.70), 330 $\left[(\mathrm{M}+1-\mathrm{F})^{+}, 41.51\right], 350\left(\mathrm{M}^{+}, 1.05\right)$.

$2,3,4,6-O$-四乙酰基- $\beta$ - $L$-吡喃型氟代甘露糖苷 $(\mathbf{3 e})$ : 得率 34\%, 无色油状物; ${ }^{1} \mathrm{H} \mathrm{NMR}\left(\mathrm{CDCl}_{3}, 300 \mathrm{MHz}\right) \delta$ : 2.06 (s, 3H, $\left.\mathrm{COCH}_{3}\right), 2.09$ (s, 3H, $\left.\mathrm{COCH}_{3}\right), 2.11(\mathrm{~s}, 3 \mathrm{H}$, $\left.\mathrm{COCH}_{3}\right), 2.16\left(\mathrm{~s}, 3 \mathrm{H}, \mathrm{COCH}_{3}\right), 4.08 \sim 4.35(\mathrm{~m}, 3 \mathrm{H}, \mathrm{H}-5$, H-6), $5.12 \sim 5.18(\mathrm{~m}, 2 \mathrm{H}, \mathrm{H}-3, \mathrm{H}-4), 5.30 \sim 5.18(\mathrm{~m}, 1 \mathrm{H}$,
$\mathrm{H}-2), 5.55(\mathrm{dd}, J=51.1,1.5 \mathrm{~Hz}, 1 \mathrm{H}, \mathrm{H}-1) ;{ }^{19} \mathrm{~F}$ NMR $\left(\mathrm{CDCl}_{3}, 282 \mathrm{MHz}, \mathrm{CF}_{3} \mathrm{COOH}\right.$ 作内标) $\delta:-65.2(\mathrm{dd}, J=$ 51.1, $10.4 \mathrm{~Hz}, 1 \mathrm{~F}, \mathrm{~F}-1$ ); IR (neat) $v: 1749,1639,1435$, 1371, 1220, 1093, $1052 \mathrm{~cm}^{-1}$; EI-MS m/z: 43 (100.00), 109 (20.08), 169 (59.20), 211 (7.88), 270 (6.79), 330 $\left[(\mathrm{M}+1-\mathrm{F})^{+}, 52.36\right], 350\left(\mathrm{M}^{+}, 0.23\right)$.

\section{References}

1 Ariki, M.; Fukui, T. J. Biochem. 1975, 78, 1191.

2 Tsuchiya, T. Adv. Carbohydr. Chem. Biochem. 1990, 48, 91.

3 Mukaiyama, T.; Murai, Y.; Shoda, S. Chem. Lett. 1981, 10, 431.

4 Hayashi, M.; Hashimoto, S.; Norori, R. Chem. Lett. 1984, 13, 1747.

5 Crich, D.; Lim, L. B. L. J. Chem. Soc. Perkin Trans. 1 1991, 2209.

6 Matsumoto, T.; Maeta, H.; Suzuki, K.; Tsuchihashi, G. Tetrahedron Lett. 1988, 29, 3567.

7 Caddick, S.; Gazzard, L.; Motherwell, W. B.; Wilkinson, J. A. Tetrahedron 1996, 52, 149.

8 Kobayashi, S.; Yoneda, A.; Fukuhara, T.; Hara, S. Tetrahedron Lett. 2004, 45, 1287.

9 Bennua-Skalmowski, B.; Vorbruggen, V. Tetrahedron Lett. 1995, 36, 2611.

10 Zhen, Z.; Tian, W. S.; Liao, Q. J. Tetrahedron Lett. 1996, 37, 8553 .

11 Tian, W. S.; Lei, Z.; Chen, L.; Huang, Y. J. Fluorine Chem. 2000, 101, 305.

12 (a) Chen, L.; Ding, K.; Tian, W. S. Chem. Commun. 2003, 838.

(b) Ding, K.; Sun, Y. S.; Tian, W. S. J. Org. Chem. 2011, 76, 1495.

(c) Sun, Y. S.; Ding, K.; Tian, W. S. Chem. Commun. 2011, 10437.

13 He, L.; Xiang, H.; Zhang, L. Y.; Tian, W. S.; He, H. L. Drug Dev. Res. 2005, 64, 203.

14 (a) Yan, Z. H.; Wang, J. Q.; Tian, W. S. Tetrahedron Lett. 2003, 44, 9383.

(b) Yan, Z. H.; Wei, M.; Tian, W. S.; Guan, C. B. Acta Chim. Sinica 2011, 69, 1269 (in Chinese).

(严兆华, 魏萌, 田伟生, 官程波, 化学学报, 2011，69, 1269.)

15 Yan, Z. H.; Tian, W. S. Tetrahedron Lett. 2004, 45, 2211.

16 Yan, Z. H.; Tian, W. S.; Zeng, F. R.; Dai, Y. F. Tetrahedron Lett. 2009, 50, 2727.

17 Tian, W. S.; Shi, Y. Prog. Chem. 2010, 22, 537 (in Chinese).

(田伟生, 史勇, 化学进展, 2010, 22, 537.)

18 Hall, L. D.; Manville, J. F. Can. J. Chem. 1969, 47, 23.

19 Hall, L. D.; Manville, J. F. Carbohydr. Res. 1967, 4, 512.

20 Hall, L. D.; Manville, J. F. Can. J. Chem. 1969, 47, 1.

21 Miethchen, R.; Kolp, G. J. Fluorine Chem. 1993, 60, 49.

(A1112162 Cheng, B.; Lu, Z.) 